\title{
Article
}

\section{Long-Term Outcomes of Personalized Stereotactic Ablative Brachytherapy for Recurrent Head and Neck Adenoid Cystic Carcinoma after Surgery or External Beam Radiotherapy: A 9-Year Study}

\author{
Yi Chen ${ }^{1,{ }^{+}}$, Jinzhao Dai ${ }^{2,+}$, Yuliang Jiang ${ }^{1}$, Zhe Ji ${ }^{1}$, Ping Jiang ${ }^{1}$, Haitao Sun ${ }^{1}$, Fei Xu ${ }^{1}$ and Junjie Wang ${ }^{1, *(D)}$ \\ 1 Department of Radiation Oncology, Peking University Third Hospital, Beijing 100191, China; \\ yichen@bjmu.edu.cn (Y.C.); yuliangjiang@bjmu.edu.cn (Y.J.); aschoff@bjmu.edu.cn (Z.J.); \\ jiangping@bjmu.edu.cn (P.J.); sunht_puth@163.com (H.S.); xuf_puth@163.com (F.X.) \\ 2 Department of Nuclear Medicine, Qingdao Central Hospital, Qingdao 266300, China; daijz_sdqd@163.com \\ * Correspondence: junjiewang@pku.edu.cn; Tel.: +86-137-0107-6310; Fax: +86-010-8226-5968 \\ + These authors contributed equally to this work.
}

Citation: Chen, Y.; Dai, J.; Jiang, Y.; Ji, Z.; Jiang, P.; Sun, H.; Xu, F.; Wang, J. Long-Term Outcomes of Personalized Stereotactic Ablative Brachytherapy for Recurrent Head and Neck

Adenoid Cystic Carcinoma after Surgery or External Beam Radiotherapy: A 9-Year Study. J. Pers. Med. 2021, 11, 839. https://doi.org/ 10.3390/jpm11090839

Academic Editors: Elisa Placidi and Ángeles Rovirosa

Received: 14 July 2021

Accepted: 22 August 2021

Published: 26 August 2021

Publisher's Note: MDPI stays neutral with regard to jurisdictional claims in published maps and institutional affiliations.

Copyright: (c) 2021 by the authors. Licensee MDPI, Basel, Switzerland. This article is an open access article distributed under the terms and conditions of the Creative Commons Attribution (CC BY) license (https:/ / creativecommons.org/licenses/by/ $4.0 /)$.

\begin{abstract}
The management of recurrent head and neck adenoid cystic carcinoma (HNACC) remains a problematic challenge. This study aims to evaluate the long-term outcomes of personalized stereotactic ablative brachytherapy (SABT) as a salvage treatment for recurrent HNACC after surgery or external beam radiotherapy (EBRT). 21 patients with recurrent HNACC after surgery or EBRT successfully underwent iodine-125 (I-125) seed SABT from May 2011 to November 2019. The objective response rate (ORR), disease control rate (DCR), local control time (LCT), overall survival (OS), symptomatic relief and adverse events (AEs) were analyzed. Following SABT, the ORR and DCR were $85.7 \%$ and $100 \%$, respectively. The $3-$, and 5 -year LCT rates were $68.8 \%$ and $55.1 \%$, respectively, and the 3 - and 5-year OS rates were $85.9 \%$ and $66.2 \%$, respectively. Furthermore, univariate analyses showed that higher D90 ( $>137.1 \mathrm{~Gy})$ was a strong positive prognostic factor of LCT $(p<0.05)$. The pain disappeared in one patient 3 months after SABT and partial pain improvement was observed in nine patients 1 to 6 months after SABT. Additionally, dyspnea was relieved in one patient with the tumor involving the trachea. The major AEs were mild intraoperative hemorrhage and skin/mucosal toxicities which were generally graded $\leq 2$ and well-tolerated. Personalized SABT was an effective and safe alternative option for recurrent HNACC after the previous failure of surgery or EBRT. The parameter of D90 may influence the local control.
\end{abstract}

Keywords: adenoid cystic carcinoma; head and neck neoplasm; Iodine-125; stereotactic ablative brachytherapy; interstitial brachytherapy

\section{Introduction}

Head and neck adenoid cystic carcinoma (HNACC) is a rare cancer, accounting for approximately $1 \%$ of all head and neck malignancies. It usually arises from the major and the minor salivary glands [1], also arising from other sites including the nasal and paranasal sinuses, trachea and larynx, lacrimal and ceruminous glands [2].

The primary therapy for HNACC remains wide surgical resection followed by adjuvant postoperative radiotherapy that significantly improves the survival [3-5]. Unfortunately, clinicians assume that "cure is probably impossible for HNACC" due to its high risk of local recurrence and distant metastasis. Even though occasional favorable survival outcomes are reported (5-year overall survival (OS) rate of $92 \%$ in the Australia study [6], 10-year OS rates of 63.7\% in the Japan study [7] and 20-year OS rate of $40 \%$ in the England study [8]), the survival rates continue to decline, even until 30 years.

Repeated local recurrence, actually, is the leading cause of treatment failure in HNACC. The local recurrence rate was $54 \%$ in the Australia study [6], and it reached $100 \%$ at 30 years 
in the Jones' study [8]. To handle the local recurrence of HNACC after initial surgery and/or external beam radiotherapy (EBRT) is a problematic challenge. Re-resection is rarely an option for most patients in clinical practice due to the difficulty of complete resection, high risk of complications, functional preservation, cosmesis and patients' refusal after suffering several prior operations. Worse still, it is rather challenging to deliver high enough doses to the recurrent disease in the previously irradiated area using re-EBRT due to the dose limitation of the adjacent normal tissues. The role of chemotherapy for recurrent HNACC remains controversial. Therefore, new therapeutic strategies are urgently needed.

In response, personalized stereotactic ablative brachytherapy (SABT) using low dose rate (LDR) or high dose rate (HDR) interstitial brachytherapy may resolve this puzzle. An increasing number of studies demonstrate that SABT, characterized by delivering high doses to the tumor and well sparing the adjacent normal tissues, is an effective alternative for most recurrent cancers [9-23]. Previous studies in our center have shown that $\mathrm{SABT}$ is an effective and safe option for recurrent head and neck squamous carcinoma and soft tissue sarcoma [9,10], recurrent cervical cancer [13], recurrent retroperitoneal lymphatic metastasis [24] and rectal cancer [16]. SABT of iodine-125 (I-125) seed implantation brachytherapy is recommended by NCCN Guidelines of Rectal Cancer 2015.v2 as a salvage treatment for locally recurrent rectal carcinoma [25]. Moreover, the SABT of HDR interventional brachytherapy is also recommended by ESMO clinical guidelines as a ablative treatment for hepatocellular carcinoma [26]. This study intends to report the long-term outcomes of recurrent HNACC patients treated with I-125 seed SABT to provide more advice for the treatment of recurrent HNACC.

\section{Results}

\subsection{Patient Characteristics}

A total of 23 patients were considered for SABT over 9 years, while two patients were finally excluded: one with a high tendency of bleeding and the other with a tumor involving the facial skin and leading ulceration. Finally, the SABT was performed with the $\mathrm{CT}$ guided freehand implantation technique for eight patients. Furthermore, a CT guided and 3D printed non-coplanar template (3D-PNCT) assisted technique was employed for other 13 patients. The characteristics and SABT information of the 21 patients are shown in Table 1.

Table 1. General information for the 21 patients in this study.

\begin{tabular}{ccc}
\hline Parameter & Category & Cases (\%) \\
\hline Age & Median (range) & $56(11-75)$ \\
Sex & Male & $8(38.1)$ \\
& Female & $13(61.9)$ \\
& 60 & $1(4.8)$ \\
& 70 & $3(14.3)$ \\
Tumor size $\left(\mathrm{cm}^{3}\right)$ & 80 & $7(33.3)$ \\
& 90 & $10(47.6)$ \\
& $<3 \mathrm{~cm}$ & $9(42.9)$ \\
Prior treatment & $3-6 \mathrm{~cm}$ & $11(52.4)$ \\
& $>6 \mathrm{~cm}$ & $1(4.8)$ \\
& None & $0(0.0)$ \\
& Surgery & $17(81.0)$ \\
Prior surgery times & EBRT & $16(76.2)$ \\
& Surgery and EBRT & $12(57.1)$ \\
& 0 & $4(19.0)$ \\
& 1 & $15(71.4)$ \\
\end{tabular}


Table 1. Cont.

\begin{tabular}{ccc}
\hline Parameter & Category & Cases (\%) \\
\hline Prior radiotherapy times & 0 & $5(23.8)$ \\
& 1 & $13(61.9)$ \\
Prior cumulative radiotherapy dose & 2 & $3(14.3)$ \\
& $<60$ Gy & $1(4.8)$ \\
GTV $\left(\mathrm{cm}^{3}\right)$ & $\geq 60$ Gy & $15(71.4)$ \\
Prescription dose (Gy) & Median (range) & $25.8(4.2-63.5)$ \\
No. of needles & Median (range) & $140(90-180)$ \\
No. of Seeds & Median (range) & $9(2-19)$ \\
Seed activity (mCi) & Median (range) & $36(8-80)$ \\
D90 (Gy) & Median (range) & $0.59(0.40-0.79)$ \\
\hline
\end{tabular}

Abbreviations: EBRT, External Beam Radiotherapy; KPS, Karnofsky Performance Score; HNACC, Head and Neck Adenoid Cystic Carcinoma; GTV, Gross Tumor Volume.

\subsection{Symptom Relief}

The pain disappeared in one patient 3 months after seeding and partial pain improvement was observed in nine patients 1 to 6 months after seeding. Additionally, dyspnea was relieved in one patient with the tumor invading the trachea.

\subsection{Treatment Response}

Four patients achieved CR (Figure 1), 14 patients achieved PR, three patients achieved $\mathrm{SD}$ and no patients developed progression within 6 months after SABT with the ORR of $85.7 \%$ and the DCR of $100 \%$.
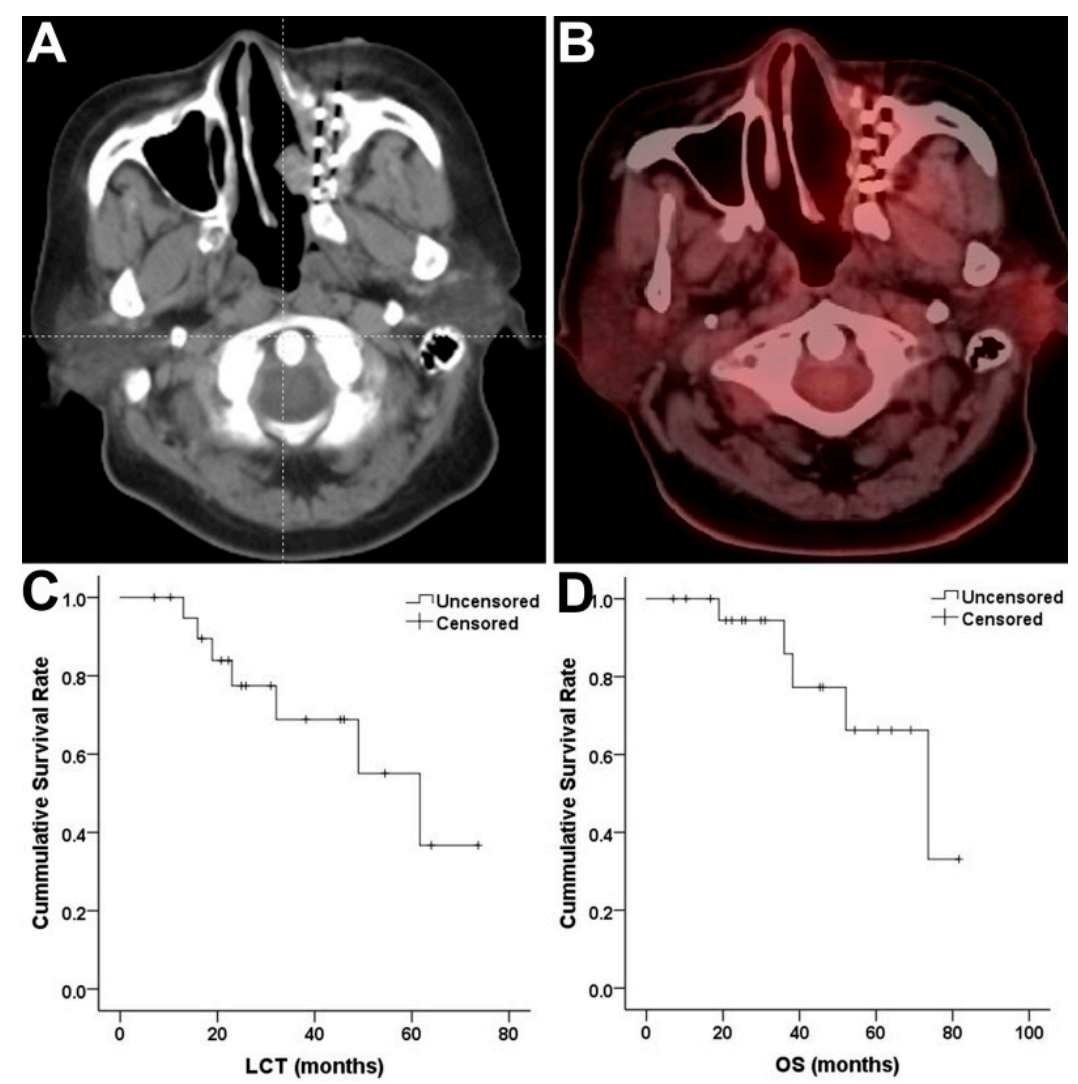

Figure 1. Short-term and long-term efficacy of stereotactic ablative brachytherapy (SABT) for recurrent head and neck adenoid cystic carcinoma (HNACC). (A,B) showed the complete response of one patient 3 months after SABT. (C,D) showed the LCT and OS of all patients. 


\section{4. $L C T$}

The median follow-up period was 37.5 months (range, 12-84 months). During the follow-up, 7 patients developed local recurrence. The median LCT was 61.7 months with three- and five-year LCT rates of $68.8 \%$ and $55.1 \%$ respectively (Figure 1 ).

\subsection{OS}

Of 21 patients, five died: one suffocated by severe hemorrhage, one died from metastasis and the other died from local progression. There was no treatment-related death. The median OS was 73.6 months with 3- and 5-year OS rates of $85.9 \%$ and $66.2 \%$, respectively (Figure 1).

\subsection{Univariate Analyses of LCT and OS}

LCT and OS were important parameters reflecting the efficacy; therefore, factors possibly influencing these two outcomes were analyzed.

Univariate analyses for LCT (Table 2) showed that the patients with higher D90 (>137.1 Gy) had a more favorable LCT than those with lower doses (61.7 months versus 32.1 months, $p=0.048$ ) (Figure 2). Multivariate analyses were not carried out due to the low event rate.

Table 2. Univariate analyses for LCT.

\begin{tabular}{|c|c|c|c|c|}
\hline \multirow{2}{*}{ Variables } & \multirow{2}{*}{ Categories } & \multicolumn{3}{|c|}{ Univariate Analyses } \\
\hline & & HR & $95 \%$ CI & $p$-Value \\
\hline \multirow{2}{*}{ Age(year) } & $>56$ & 0.480 & $0.107-2.161$ & 0.339 \\
\hline & $\leq 56$ & & & \\
\hline \multirow{2}{*}{ Sex } & Female & 1.248 & $0.270-5.761$ & 0.777 \\
\hline & Male & & & \\
\hline \multirow{2}{*}{ KPS } & $>80$ & 0.228 & $0.043-1.205$ & 0.082 \\
\hline & $\leq 80$ & & & \\
\hline \multirow{2}{*}{ Prior Surgery } & Yes & 0.774 & $0.147-4.071$ & 0.762 \\
\hline & No & & & \\
\hline \multirow{2}{*}{ Prior EBRT } & Yes & 1.587 & $0.185-13.625$ & 0.674 \\
\hline & No & & & \\
\hline \multirow{2}{*}{$\mathrm{GTV}\left(\mathrm{cm}^{3}\right)$} & $>25.8$ & 1.037 & $0.229-4.688$ & 0.963 \\
\hline & $\leq 25.8$ & & & \\
\hline \multirow{2}{*}{ D90(Gy) } & $>137.1$ & 0.117 & $0.014-0.979$ & 0.048 \\
\hline & $\leq 137.1$ & & & \\
\hline
\end{tabular}

bbreviations: LCT, Local control time; KPS, Karnofsky Performance Score; EBRT, External Beam Radiotherapy; HR, Hazard Ratio; CI, Confidence Intervals. 


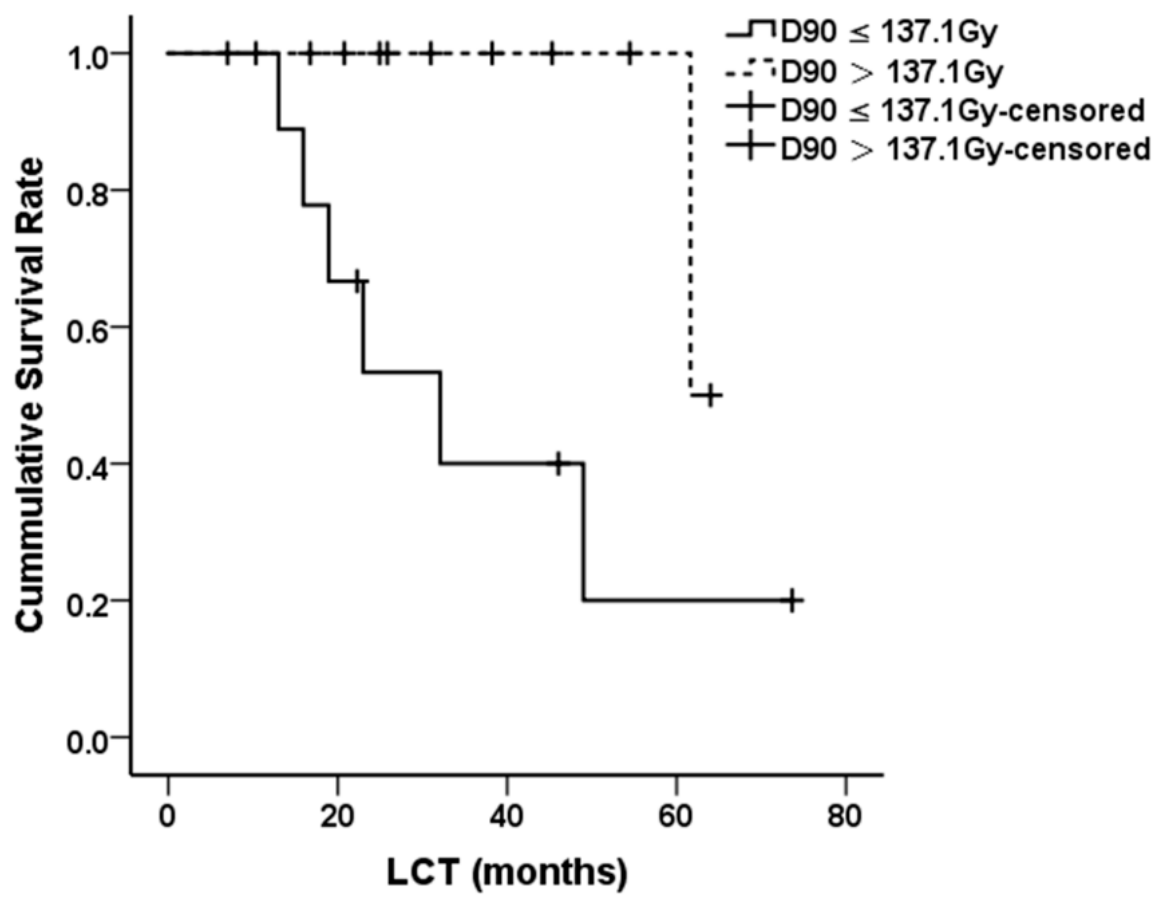

Figure 2. Local control time (LCT) of patients with different D90.

Univariate analyses indicated no independent factors for OS in this case series (Table 3).

Table 3. Univariate analyses for OS.

\begin{tabular}{|c|c|c|c|c|}
\hline \multirow{2}{*}{ Variables } & \multirow{2}{*}{ Categories } & \multicolumn{3}{|c|}{ Univariate Analyses } \\
\hline & & HR & $95 \%$ CI & $p$-Value \\
\hline Age (year) & $\begin{array}{l}>56 \\
\leq 56\end{array}$ & 0.534 & $0.089-3.209$ & 0.493 \\
\hline Sex & $\begin{array}{c}\text { Female } \\
\text { Male }\end{array}$ & 3.290 & $0.361-30.016$ & 0.291 \\
\hline KPS & $\begin{array}{l}>80 \\
\leq 80\end{array}$ & 2.796 & $0.308-25.367$ & 0.361 \\
\hline Prior Surgery & $\begin{array}{l}\text { Yes } \\
\text { No }\end{array}$ & 0.537 & $0.073-3.940$ & 0.541 \\
\hline Prior EBRT & $\begin{array}{l}\text { Yes } \\
\text { No }\end{array}$ & 0.860 & $0.087-8.458$ & 0.897 \\
\hline $\operatorname{GTV}\left(\mathrm{cm}^{3}\right)$ & $\begin{array}{l}>25.8 \\
\leq 25.8\end{array}$ & 6.022 & $0.664-54.734$ & 0.111 \\
\hline D90(Gy) & $\begin{array}{l}>137.1 \\
\leq 137.1\end{array}$ & 0.273 & $0.030-2.453$ & 0.247 \\
\hline
\end{tabular}

Abbreviations: OS, Overall Survival; KPS, Karnofsky Performance Score; EBRT, External Beam Radiotherapy; HR, Hazard Ratio; CI, Confidence Intervals.

\subsection{AEs}

The puncture-related and radiation-related AEs were reviewed. Mild intraoperative hemorrhage was observed in all cases while no moderate and severe hemorrhage occurred during and after operation. No patient developed postoperative infection. Six patients developed Grade 1 skin reaction and one developed Grade 2 skin reaction. Grade 1 mucosal reaction was observed in two patients. 


\section{Discussion}

In this study, most of the patients (12/21) underwent surgical resection and neoadjuvant or adjuvant EBRT as an initiative therapy before recurrence. Some patients even received multiple times of resection and EBRT. It is a challenge to deal with the local recurrent disease after the failures of various therapies. Patients in the present study were deemed as unfit for surgery in the light of the surgeon's consultation or personal refusal. Additionally, re-EBRT, due to the dose limitation of the normal structures or individual choice, was not recommended.

SABT, theoretically, can deliver a high enough dose to the tumor and spare the adjacent normal tissues considering its better conformity, more rapid dose fall-off and lower dose rate compared to EBRT [27]. The facts bear out the theory. SABT has been recommended as one of the standard care methods for prostate carcinoma by the guidelines for years. Furthermore, it is also recommended by ESMO clinical guidelines as an ablative treatment for hepatocellular carcinoma [26]. Previous studies, in increasing numbers, further demonstrate its efficacy and safety in various cancers, especially for head and neck cancer after multiple therapies [9-23]. The SABT is constantly evolving, especially the invention of 3D-PNCT that significantly improves the accuracy, efficacy, security and productivity of the SABT. Therefore, SABT may be a preferred alternative for recurrent HNACC.

In this study, ORR was $85.7 \%$ and DCR was $100 \%$ in the patients treated with $\mathrm{I}-125$ seed SABT. Moreover, the median LCT reached 61.7 months with the 3- and 5-year LCT rates of $68.8 \%$ and $55.1 \%$, respectively, which indicated a good short-term efficacy of SABT guided by 3D-PNCT and/or CT. These favorable results are consistent with previous studies. Ashamalla et al., in 2002, even tried to use ${ }^{198} \mathrm{Au}$ seeds to treat head and neck cancer and reported an average progression-free survival of 52 months in recurrent HNACC (4 patients) although the seeds were implanted with freehand without CT guidance and template assistance [28]. Zhang et al. reported a 5-year LCT rate of $57.3 \%$ for recurrent HNACC (29 patients) [29]. In Zhang's study, seed implantation was assisted by a 3Dprinting coplanar template (3D-PCT) and guided by CT. Different from the above studies, in our study most cases of SABT were assisted by the 3D-PNCT and guided by the CT. The results from previous studies and ours suggested that SABT had a good efficacy for HNACC. Interestingly, the local control seemed better in our and Zhang's study than in Ashamalla's study, which was probably attributed to the application of template and CT. Our previous work indicated that template assistance significantly improved the accuracy and efficacy of SABT [30]. Additionally, 3D-PNCT is superior to the 3D-PCT in some aspects. Ji et al. reported that the dose in target volume margin was higher, needles were fewer, and safety was higher in the plan assisted by 3D-PNCT than in the plan assisted by 3D-PCT for lung cancer [31]. Qu et al. pointed out that both 3D-PNCT and 3D-PCT plans for patients with pelvic recurrence of gynecological malignancies had similar dosimetry, but 3D-PNCT improved the security of SABT [32]. This study, as far as we know, is probably the first to report the long-term outcomes of the I-125 seed SABT assisted by 3D-PNCT and CT for recurrent HNACC after the failure of previous surgery and/or EBRT. As to long-term survival, our study showed that the median OS was 73.6 months with the 3- and 5 -year OS rates of $85.9 \%$ and $66.2 \%$, respectively. Zhang et al. reported similar favorable results [29].

As the therapeutic experience of the recurrent HNACC is scarce, prognostic factors remain unclear. Univariate analyses showed that the LCT was significantly prolonged in patients with a higher target dose (D90 > 137.1 Gy), which suggested that the local control may be improved if the prescribed dose is raised. Ji et al. [9] and Qu et al. [13] have reported similar results in recurrent head and neck cancer and cervical cancer. Our previous work reconfirmed this in retroperitoneal lymph node metastasis [24]. Therefore, exploring an appropriate prescribed dose of SABT for recurrent HNACC is of crucial clinical significance. 
Most of the patients in this study obtained pain relief after implantation. In addition, one patient was relieved of severe dyspnea. Similar results are observed in previous studies [29]. These consistent results suggest that SABT is effective in symptom relief.

The only recorded AEs were mild intraoperative hemorrhage and skin/mucosal toxicities generally graded $\leq 2$ and well-tolerated. Previous studies also observed low toxicities of SABT as a salvage treatment for recurrent head and neck cancer. Ji et al. reported that grade $3 \mathrm{skin} /$ mucosal toxicities were $7.9 \%$ and grade 4 were $2 \%$. Jiang et al. [33] explored the side effects of SABT in the treatment of recurrent head and neck cancer showing that $14.4 \%$ of patients had grade $1 / 2 \mathrm{skin} /$ mucosal toxicities, while no patients suffered from grade $3 / 4 \mathrm{skin} /$ mucosal reaction. Bussu et al. reported that transient paralysis of the sixth cranial nerve was the only recorded AE in head and neck cancer treated with SABT of HDR interstitial brachytherapy [19]. Strnad et al. reported that the soft tissue or bone necrosis rates were $17.3 \%$ and $9.6 \%$, respectively, in head and neck malignancies (only $3 \%$ of the cases needed surgical treatment) after SABT with/without other therapeutic modalities [21]. Teudt and Kovacs et al. reported that the safety of perioperative SABT in recurrent or advanced head and neck metastases was good (the acute and late toxicities were both graded $\leq 2$ ) [23]. Conversely, the toxicities of re-EBRT seemed more severe in head and neck malignancies compared to SABT. De Crevoisier et al. [34] reported that the Grade 3 and Grade 4 mucositis were 32\% and 14\%, respectively, for re-EBRT in head and neck carcinoma. Furthermore, RTOG 9610 reported that the patients treated with re-EBRT combined with chemotherapy developed grade $3 \mathrm{skin} /$ mucosal toxicities in $34.2 \%$ of the patients and grade $4 \mathrm{skin} /$ mucosal toxicities in $8 \%$ of the patients [35]. Generally, the AEs were of lower grade and well-tolerated, indicating the safe nature of SABT.

As to limitations, the retrospective nature of this study and limited sample size, partly due to the rare morbidity, lowered the evidence level of this study. Even so, it provided preliminary evidence for SABT as a means to treat recurrent HNACC after surgery or EBRT. The evidence from prospective studies is warranted to further confirm the role of SABT in recurrent HNACC.

\section{Materials and Methods}

\subsection{Patients}

The patients diagnosed with recurrent HNACC after surgery or EBRT, undergoing I-125 seed SABT from May 2011 to November 2019, were retrospectively reviewed. Written informed consent was obtained from each patient and this study was approved by the Ethics Committee of our hospital.

\subsection{Inclusion and Exclusion Criteria}

The inclusion criteria are as follows: (1) confirmed HNACC by pathologic or imaging diagnosis; (2) disease $>1 \mathrm{~cm}$ and $\leq 7 \mathrm{~cm}$; (3) medically inoperable or individual refusal to resection; (4) unfit for re-EBRT after a comprehensive evaluation by radiation oncologists or individual refusal to re-EBRT; (5) tolerate anesthesia and puncture; (6) suitable puncture access; (7) no severe tendency for bleeding; (8) Karnofsky performance status (KPS) $\geq 60$; (9) adequate hematological reserves: white blood cell counts $\geq 3 \times 109 / \mathrm{mL}$; granulocyte counts $\geq 1.5 \times 109 / \mathrm{mL}$; hemoglobin $\geq 90 \mathrm{~g} / \mathrm{L}$; platelet $>50 \times 109 / \mathrm{L} ;(10)$ adequate hepatic function: total serum bilirubin concentration $\leq 1.5$ times the upper limit normal (ULN); serum transaminases and alkaline phosphatase $\leq 2.5 \mathrm{ULN}$; (11) adequate renal function: serum creatinine concentration $\leq 1.5 \mathrm{ULN}$ or creatinine clearance $>50 \mathrm{~mL} / \mathrm{min}$; (12) adequate heart function: left ventricular ejection fraction (LVEF) $\geq 50 \%$; (13) expected survival $>3$ months.

The exclusion criteria are as follows: (1) unconfirmed head and neck mass; (2) any mental disorder; (3) anticoagulant or anti-aggregate therapies were discontinued for less than 1 week before SABT; (4) patients with active infectious disease, trauma, stroke and severe wounds; (5) other somatic comorbidities of clinical concern; (6) pregnancy and lactation, (7) patient consent withdrawal. 


\subsection{Treatment}

The personalized SABT procedure mainly includes individualized preoperative treatment planning, intraoperative seed implantation and postoperative dosimetric evaluation (Figure 3).

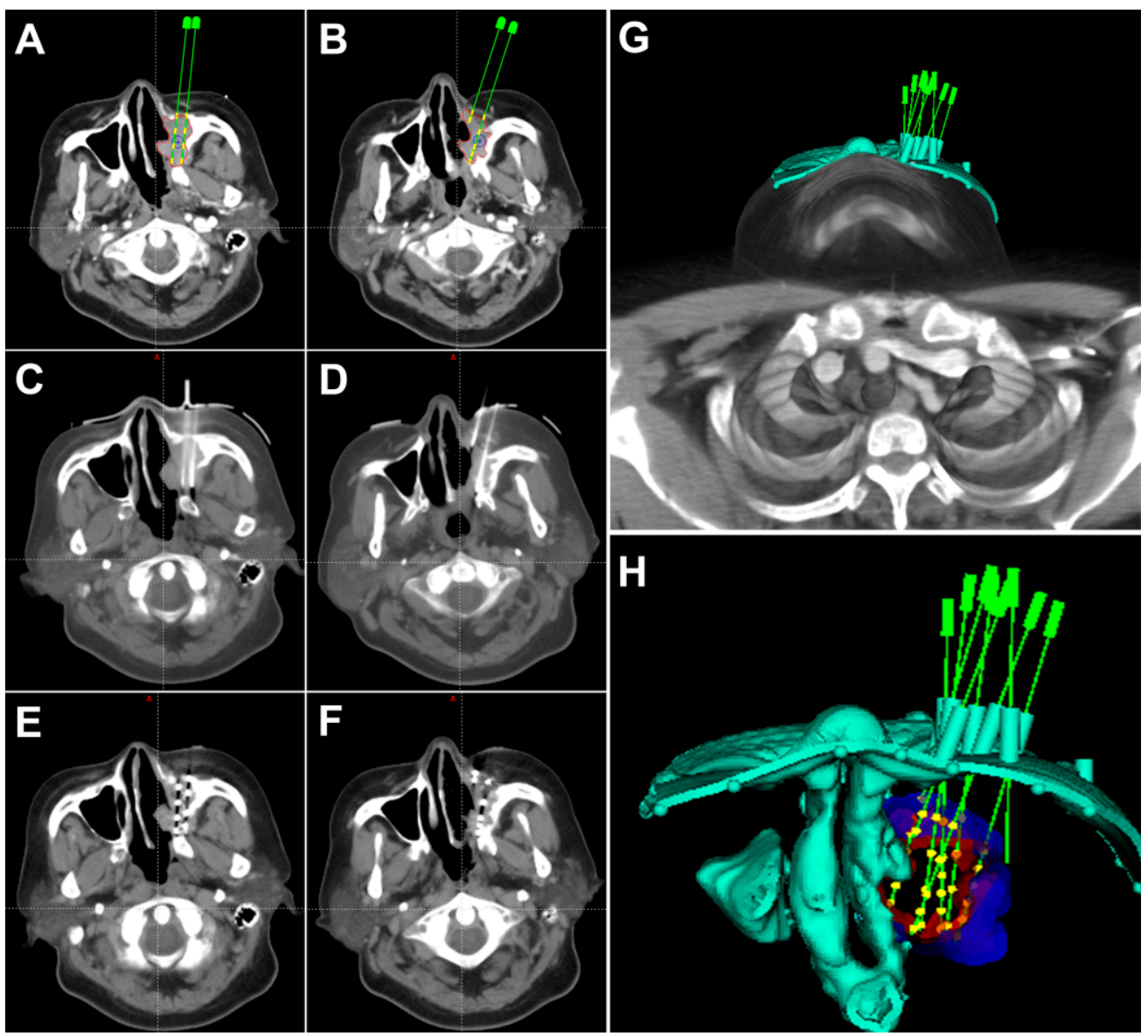

Figure 3. The CT images of pre-, intra- and post-operation. $(\mathbf{A}, \mathbf{B})$ show the preoperative treatment planning including the planned locations of needles, distribution of seeds and gross tumor volume (GTV). The green needles and yellow seeds are simulated needles and seeds in the brachytherapy treatment planning system (B-TPS), respectively. (C,D) show the actual locations of needles during operation. $(\mathrm{E}, \mathrm{F})$ show the actual postoperative distribution of seeds in target volume. $(\mathbf{G}, \mathbf{H})$ show the 3D-printing non-coplanar template (3D-PNCT) model with guide holes in the B-TPS.

\subsection{Preoperative Individualized Treatment Planning}

Patients undergo setup and CT simulation 2-3 days prior to the seed implantation to obtain three-dimensional information of tumor volume and organs at risk (OARs). Then we use the brachytherapy treatment planning system (B-TPS; Beijing Feitian Industries Inc. and Beijing University of Aeronautics and Astronautics, Beijing, China) to evaluate the feasibility of seeding. If feasible, the pretreatment plan is made by a medical physicist including the delineation of gross tumor volume (GTV) and adjacent OARs, determination of prescription dose, design of the accesses of the insertion needles (direction, distribution and depth) and spatial distribution of I-125 seeds, and calculation of the dose in the GTV and OARs. The dosimetric goal is that the dose received by $90 \%$ of the GTV (GTV D90) reaches the prescription dose as far as possible. The doses delivered to the OARs are as low as possible through optimization. 


\subsection{Intraoperative Implantation}

Two different methods are used for intraoperative implantation. Method A is used for the patients before 2015 and method B is used for the patients after 2015 when the 3D printed non-coplanar template (3D-PNCT) was invented by our center (Patent No. ZL201620414011.9).

Method A: Implantation guided by CT without the assistance of the template. After re-setup, skin preparation, draping and local anesthesia, disposable needles (Mick Radio Nuclear Instruments, Mount Vernon, NY, USA) are inserted into the target volume under $\mathrm{CT}$ guidance along the puncture points on the skin which are designed preoperatively. I-125 seeds (CIAE-6711; Chinese Atomic Energy Science Institution, Beijing, China) are then implanted by the Mick applicator (Mick Radio-Nuclear Instruments Inc., Mount Vernon, NY, USA).

Method B: Implantation guided by CT with aid of the 3D-PNCT. After the treatment plan is designed in the B-TPS, a 3D personal template model is generated at once including the information of needle distribution and the characteristics of the therapeutic area outline (Figure 3G,H). The 3D-PNCT is then printed by the 3D light-cured rapid-forming printer. Then after re-setup, skin preparation, draping and local anesthesia, the 3D-PNCT is aligned to the surface of the therapeutic area according to the outline of the patient, positioning line on the patient, alignment line on the 3D-PNCT and laser. Then the implantation needles are inserted to the predetermined depth through the guide holes on the 3D-PNCT guided by CT. "Fine-tuning", if necessary, is carried out. After insertion, the I-125 seeds are implanted into the tumor as mentioned above.

See our previous work [10] for the details of the seeding procedures.

\subsection{Postoperative Evaluation}

Following seed implantation, a CT scan is immediately conducted to validate the postoperative distribution of seeds. Then the images are loaded to B-TPS to calculate the actual seeds and dose distribution (Figure $3 \mathrm{E}, \mathrm{F}$ ). Dosimetric parameters including D90, D100, V100, V150, V200 are used to evaluate the dosimetry, where Dx indicates the dose delivered to $\mathrm{x} \%$ of GTV, and Vx indicates the percentage of GTV receiving $\mathrm{x} \%$ of the prescribed dose.

All physicians participating in the procedure of SABT are well trained and legally qualified. All procedures are performed in accordance with relevant guidelines and regulations.

\subsection{Follow-Up}

Patients are followed up every 3 months for the first 2 years, then every 6 months from 3 to 5 years and annually thereafter.

\subsection{Clinical Outcomes}

The clinical outcomes included the objective response rate (ORR), disease control rate (DCR), local control time (LCT), overall survival (OS), safety and symptom relief. Response Evaluation Criteria in Solid Tumors (RECIST) version 1.1 is used to evaluate the clinical responses including complete response (CR), partial response (PR), stable disease (SD), and progressive disease (PD). ORR is confirmed in cases with CR or PR and DCR is confirmed in cases with CR or PR or SD. LCT is defined as the time from SABT to local progression with OS defined as the time from SABT to death from any cause. The safety profiles include operation complications and radiation-related adverse events (AEs) assessed as per RTOG Common Toxicity Criteria.

\subsection{Statistical Analysis}

The parameters are expressed as categorical variables or continuous variables. The categorical variables are compared by the Chi-square or Fisher's exact test, while continuous variables are compared by the t-test or rank-sum test. The LCT and OS are analyzed through the Kaplan-Meier method and compared using the log-rank test. Univariate anal- 
yses of LCT and OS are performed using the Cox proportional hazard regression model. $p<0.05$ is considered as statistically significant. SPSS 24.0 software (SPSS, Chicago, IL, USA) is used for the statistical analyses.

\section{Conclusions}

For patients with recurrent HNACC after the failure of surgery and EBRT, SABT showed a good response rate, favorable local control and survival in accompany with a satisfying safety, which suggested that SABT can be an effective and safe alternative option for recurrent HNACC. The dose may influence the local control, and dose constraints could be defined in larger patient cohorts.

Author Contributions: Conceptualization, J.W.; Methodology, P.J;; Formal Analysis, Y.C.; Investigation, Y.J., J.D. and H.S.; Resources, J.W. and H.S.; Data Curation, Y.J. and J.D.; Writing-Original Draft Preparation, Y.C.; Writing—Review \& Editing, Y.J., Z.J., P.J. and J.W.; Supervision, F.X.; Project Administration, J.W.; Funding Acquisition, J.W. All authors have read and agreed to the published version of the manuscript.

Funding: This work was supported by National Key Research and Development Program of China (2019YFB1311300).

Institutional Review Board Statement: The study was conducted according to the guidelines of the Declaration of Helsinki, and approved by the Institutional Ethics Committee of Peking University Third Hospital (protocol code IRB00006761-M20210702 and July 2nd).

Informed Consent Statement: Informed consent was obtained from all subjects involved in the study.

Data Availability Statement: The data presented in this study are available on request from the corresponding author. The data are not publicly available due to privacy or ethical restrictions.

Acknowledgments: We are grateful to Edwin Leong for his help with editing and improvements of this manuscript. (Ethical No. IRB00006761-M2019226).

Conflicts of Interest: The authors declare no conflict of interest.

\section{References}

1. Vander, P.V.; Balm, A.J.; Hilgers, F.J.; Tan, I.B.; Loftus-Coll, B.M.; Keus, R.B.; Hart, A.A. Prognostic factors for long term results of the treatment of patients with malignant submandibular gland tumors. Cancer 1999, 85, 2255-2264. [CrossRef]

2. Coca-Pelaz, A.; Rodrigo, J.P.; Bradley, P.J.; Vander, P.V.; Triantafyllou, A.; Hunt, J.L.; Strojan, P.; Rinaldo, A.; Haigentz, M.J.; Takes, R.P.; et al. Adenoid cystic carcinoma of the head and neck-An update. Oral Oncol. 2015, 51, 652-661. [CrossRef]

3. Chen, A.M.; Bucci, M.K.; Weinberg, V.; Garcia, J.; Quivey, J.M.; Schechter, N.R.; Phillips, T.L.; Fu, K.K.; Eisele, D.W. Adenoid cystic carcinoma of the head and neck treated by surgery with or without postoperative radiation therapy: Prognostic features of recurrence. Int. J. Radiat. Oncol. Biol. Phys. 2006, 66, 152-159. [CrossRef]

4. Mendenhall, W.M.; Morris, C.G.; Amdur, R.J.; Werning, J.W.; Hinerman, R.W.; Villaret, D.B. Radiotherapy alone or combined with surgery for adenoid cystic carcinoma of the head and neck. Head Neck 2004, 26, 154-162. [CrossRef]

5. Prokopakis, E.P.; Snyderman, C.H.; Hanna, E.Y.; Carrau, R.L.; Johnson, J.T.; D'Amico, F. Risk factors for local recurrence of adenoid cystic carcinoma: The role of postoperative radiation therapy. Am. J. Otolaryngol. 1999, 20, 281-286. [CrossRef]

6. DeAngelis, A.F.; Tsui, A.; Wiesenfeld, D.; Chandu, A. Outcomes of patients with adenoid cystic carcinoma of the minor salivary glands. Int. J. Oral Maxillofac. Surg. 2011, 40, 710-714. [CrossRef] [PubMed]

7. Ishida, E.; Ogawa, T.; Rokugo, M.; Ishikawa, T.; Wakamori, S.; Ohkoshi, A.; Usubuchi, H.; Higashi, K.; Ishii, R.; Nakanome, A.; et al. Management of adenoid cystic carcinoma of the head and neck: A single-institute study with over 25-year follow-up. Head Face Med. 2020, 16, 14. [CrossRef] [PubMed]

8. Jones, A.S.; Hamilton, J.W.; Rowley, H.; Husband, D.; Helliwell, T.R. Adenoid cystic carcinoma of the head and neck. Clin. Otolaryngol. Allied Sci. 1997, 22, 434-443. [CrossRef] [PubMed]

9. Ji, Z.; Jiang, Y.; Tian, S.; Guo, F.; Peng, R.; Xu, F.; Sun, H.; Fan, J.; Wang, J. The Effectiveness and Prognostic Factors of CT-Guided Radioactive I-125 Seed Implantation for the Treatment of Recurrent Head and Neck Cancer after External Beam Radiation Therapy. Int. J. Radiat. Oncol. Biol. Phys. 2019, 103, 638-645. [CrossRef]

10. Chen, Y.; Jiang, Y.; Ji, Z.; Jiang, P.; Xu, F.; Zhang, Y.; Guo, F.; Peng, R.; Li, X.; Sun, H.; et al. Efficacy and safety of CT-guided (125)I seed implantation as a salvage treatment for locally recurrent head and neck soft tissue sarcoma after surgery and external beam radiotherapy: A 12-year study at a single institution. Brachytherapy 2020, 19, 81-89. [CrossRef] 
11. Ji, Z.; Jiang, Y.; Guo, F.; Peng, R.; Sun, H.; Fan, J.; Xu, F.; Wang, J. Safety and efficacy of CT-guided radioactive iodine-125 seed implantation assisted by a 3D printing template for the treatment of thoracic malignancies. J. Cancer Res. Clin. Oncol. 2020, 146, 229-236. [CrossRef]

12. Jia, S.N.; Wen, F.X.; Gong, T.T.; Li, X.; Wang, H.J.; Sun, Y.M.; Yang, Z.C. A review on the efficacy and safety of iodine-125 seed implantation in unresectable pancreatic cancers. Int. J. Radiat. Biol. 2020, 96, 383-389. [CrossRef] [PubMed]

13. Qu, A.; Jiang, P.; Sun, H.; Jiang, W.; Jiang, Y.; Tian, S.; Wang, J. Efficacy and dosimetry analysis of image-guided radioactive 125-I seed implantation as salvage treatment for pelvic recurrent cervical cancer after external beam radiotherapy. J. Gynecol. Oncol. 2019, 30, e9. [CrossRef] [PubMed]

14. Wang, J.; Zhang, F.; Guo, J.; Chai, S.; Zheng, G.; Zhang, K.; Liao, A.; Jiang, P.; Jiang, Y.; Ji, Z. Expert consensus workshop report: Guideline for three-dimensional printing template-assisted computed tomography-guided (125)I seeds interstitial implantation brachytherapy. J. Cancer Res. Ther. 2017, 13, 607-612. [CrossRef]

15. Wang, J.; Chai, S.; Zheng, G.; Jiang, Y.; Ji, Z.; Guo, F.; Zhuang, H.; Zhang, K. Expert consensus statement on computed tomography-guided (125)I radioactive seeds permanent interstitial brachytherapy. J. Cancer Res. Ther. 2018, 14, 12-17. [CrossRef] [PubMed]

16. Wang, J.J.; Yuan, H.S.; Li, J.N.; Jiang, Y.L.; Tian, S.Q.; Yang, R.J. CT-guided radioactive seed implantation for recurrent rectal carcinoma after multiple therapy. Med. Oncol. 2010, 27, 421-429. [CrossRef] [PubMed]

17. Yao, L.; Jiang, Y.; Jiang, P.; Wang, H.; Meng, N.; Qu, A.; Tian, S.; Sun, H.; Liu, C.; Wang, J.; et al. CT-guided permanent 125I seed interstitial brachytherapy for recurrent retroperitoneal lymph node metastases after external beam radiotherapy. Brachytherapy 2015, 14, 662-669. [CrossRef]

18. Wang, J.; Yuan, H.; Ma, Q.; Liu, X.; Wang, H.; Jiang, Y.; Tian, S.; Yang, R. Interstitial 125I seeds implantation to treat spinal metastatic and primary paraspinal malignancies. Med. Oncol. 2010, 27, 319-326. [CrossRef]

19. Bussu, F.; Tagliaferri, L.; Mattiucci, G.; Parrilla, C.; Rizzo, D.; Gambacorta, M.A.; Lancellotta, V.; Autorino, R.; Fonnesu, C.; Kihlgren, C.; et al. HDR interventional radiotherapy (brachytherapy) in the treatment of primary and recurrent head and neck malignancies. Head Neck 2019, 41, 1667-1675. [CrossRef]

20. Narayana, A.; Cohen, G.N.; Zaider, M.; Chan, K.; Lee, N.; Wong, R.J.; Boyle, J.; Shaha, A.; Kraus, D.; Shah, J.; et al. High-dose-rate interstitial brachytherapy in recurrent and previously irradiated head and neck cancers-Preliminary results. Brachytherapy 2007, 6, 157-163. [CrossRef]

21. Strnad, V.; Lotter, M.; Kreppner, S.; Fietkau, R. Reirradiation for recurrent head and neck cancer with salvage interstitial pulsed-dose-rate brachytherapy: Long-term results. Strahlenther. Onkol. 2015, 191, 495-500. [CrossRef] [PubMed]

22. Tagliaferri, L.; Bussu, F.; Fionda, B.; Catucci, F.; Rigante, M.; Gambacorta, M.A.; Autorino, R.; Mattiucci, G.C.; Miccichè, F.; Placidi, E.; et al. Perioperative HDR brachytherapy for reirradiation in head and neck recurrences: Single-institution experience and systematic review. Tumori 2017, 103, 516-524. [CrossRef] [PubMed]

23. Teudt, I.U.; Kovàcs, G.; Ritter, M.; Melchert, C.; Soror, T.; Wollenberg, B.; Meyer, J.E. Intensity modulated perioperative HDR brachytherapy for recurrent and/or advanced head and neck metastases. Eur. Arch. Otorhinolaryngol. 2016, 273, $2707-2715$. [CrossRef] [PubMed]

24. Chen, Y.; Jiang, Y.; Ji, Z.; Jiang, P.; Xu, F.; Zhang, Y.; Zhang, P.; Guo, F.; Li, X.; Sun, H.; et al. Dosimetry, efficacy, and safety of three-dimensional printing noncoplanar template-assisted and CT-guided 125I seed implantation for recurrent retroperitoneal lymphatic metastasis after external beam radiotherapy. Brachytherapy 2020, 19, 380-388. [CrossRef] [PubMed]

25. Benson, A.R.; Venook, A.P.; Bekaii-Saab, T.; Chan, E.; Chen, Y.J.; Cooper, H.S.; Engstrom, P.F.; Enzinger, P.C.; Fenton, M.J.; Fuchs, C.S.; et al. Rectal Cancer, Version 2.2015. J. Natl. Compr. Canc. Netw. 2015, 13, 719-728. [CrossRef]

26. Vogel, A.; Cervantes, A.; Chau, I.; Daniele, B.; Llovet, J.M.; Meyer, T.; Nault, J.C.; Neumann, U.; Ricke, J.; Sangro, B.; et al. Hepatocellular carcinoma: ESMO Clinical Practice Guidelines for diagnosis, treatment and follow-up. Ann. Oncol. 2018, 29, v238-v255. [CrossRef]

27. Rosenblatt, E.; Meushar, N.; Eidelman, M.; Kuten, A. Low dose-rate interstitial brachytherapy in soft tissue sarcomas. Sarcoma 1999, 3, 101-105. [CrossRef]

28. Ashamalla, H.; Rafla, S.; Zaki, B.; Ikoro, N.C.; Ross, P. Radioactive gold grain implants in recurrent and locally advanced head-and-neck cancers. Brachytherapy 2002, 1, 161-166. [CrossRef]

29. Huang, M.W.; Zheng, L.; Liu, S.M.; Shi, Y.; Zhang, J.; Yu, G.Y.; Zhang, J.G. 125I brachytherapy alone for recurrent or locally advanced adenoid cystic carcinoma of the oral and maxillofacial region. Strahlenther. Onkol. 2013, 189, 502-507. [CrossRef]

30. Wang, L.; Wang, H.; Jiang, Y.; Ji, Z.; Guo, F.; Jiang, P.; Qiu, B.; Sun, H.; Fan, J.; Li, W.; et al. Dosimetric comparison of computed tomography-guided iodine-125 seed implantation assisted with and without three-dimensional printing non-coplanar template in locally recurrent rectal cancer: A propensity score matching study. J. Contemp. Brachyther. 2021, 13, 18-23. [CrossRef]

31. Ji, Z.; Sun, H.; Jiang, Y.; Guo, F.; Peng, R.; Fan, J.; Wang, J. Comparative study for CT-guided (125)I seed implantation assisted by 3D printing coplanar and non-coplanar template in peripheral lung cancer. J. Contemp. Brachyther. 2019, 11, 169-173. [CrossRef] [PubMed]

32. Qu, A.; Wang, J.J.; Jiang, Y.L.; Sun, H.T.; Jiang, P.; Ji, Z.; Guo, F.X.; Fan, J.H.; Li, W.Y. Comparison of preoperative planning of radioactive seed implantation for pelvic wall recurrent gynecological malignant tumors between 3D-printing non-coplanar template and 3D-printing coplanar template. Zhonghua Yi Xue Za Zhi 2019, 99, 841-843. [PubMed] 
33. Jiang, Y.; Ji, Z.; Guo, F.; Peng, R.; Sun, H.; Fan, J.; Wei, S.; Li, W.; Liu, K.; Lei, J.; et al. Side effects of CT-guided implantation of 125I seeds for recurrent malignant tumors of the head and neck assisted by 3D printing non co-planar template. Radiat. Oncol. 2018, 13, 18. [CrossRef] [PubMed]

34. DeCrevoisier, R.; Bourhis, J.; Domenge, C.; Wibault, P.; Koscielny, S.; Lusinchi, A.; Mamelle, G.; Janot, F.; Julieron, M.; Leridant, A.M.; et al. Full-dose reirradiation for unresectable head and neck carcinoma: Experience at the Gustave-Roussy Institute in a series of 169 patients. J. Clin. Oncol. 1998, 16, 3556-3562. [CrossRef]

35. Spencer, S.A.; Harris, J.; Wheeler, R.H.; Machtay, M.; Schultz, C.; Spanos, W.; Rotman, M.; Meredith, R.; Ang, K.K. Final report of RTOG 9610, a multi-institutional trial of reirradiation and chemotherapy for unresectable recurrent squamous cell carcinoma of the head and neck. Head Neck 2008, 30, 281-288. [CrossRef] 